\title{
Aceitação da vacina contra COVID-19 entre público diagnosticado com sindrome gripal
}

COVID-19 vaccine acceptance among the public diagnosed with the flu-like syndrome Aceptación de la vacuna contra la COVID-19 entre el público diagnosticado con síndrome gripal

Telma Maria Evangelista de Araújjo ${ }^{1}$ (D) https://orcid.org/0000-0001-5628-9577

Ayla Maria Calixto de Carvalho ${ }^{2}$ iD https://orcid.org/0000-0002-7139-5383

Inês Fronteira ${ }^{3}$ ID https://orcid.org/0000-0003-1406-4585

Andréia Alves de Sena Silva ${ }^{4}$ id https://orcid.org/0000-0002-2990-8202

Karoline Alencar Rodrigues ${ }^{4}$ ic https://orcid.org/0000-0002-7150-5175

Géssica Soares Queiroz ${ }^{1}$ id https://orcid.org/0000-0001-7669-3913

Lorena Rocha de Abrantes Carcará1 io https://orcid.org/0000-0001-5273-8339

Como citar:

Araújo TM, Carvalho AM, Fronteira I, Silva AA, Rodrigues KA, Queiroz GS, et al. Aceitação

da vacina contra COVID-19 entre público diagnosticado com síndrome gripal. Acta Paul Enferm. 2021;34:eAPE000086.

DOI

http://dx.doi.org/10.37689/actaape/2021A0000086

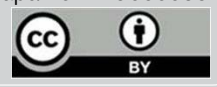

Descritores

Adesão à medicação; COVID-19; Infecções por coronavírus; Vacinas; Adulto; Saúde pública

Keywords

Medication adherence; COVID-19; Coronavirus infections; Vaccines; Adult; Public health

Descriptores

Cumplimiento de la medicación; COVID-19; Infecciones por coronavirus; Vacunas; Adulto;

Salud publica

Submetido 14 de Janeiro de 2021

Aceito 26 de Julho de 202

Autor correspondente Ayla Maria Calixto de Carvalho E-mail: aylamariacalixto@gmail.com

\section{Resumo}

Objetivo: Analisar a aceitabilidade da vacina contra COVID-19 entre pessoas com diagnóstico de síndrome gripal.

Métodos: Estudo transversal, com amostra aleatória composta por 454 participantes. Os dados foram coletados no período de março a agosto de 2020, em duas etapas: na primeira, coletaram-se os dados disponíveis nos sistemas de informação, utilizando-se instrumento adaptado da ficha de investigação de síndrome gripal suspeito de doença por COVID-19, e na segunda etapa, procedeu-se a coleta do dado primário junto ao participante, por meio de ligação telefônica. Na análise bivariada foi realizado o teste quiquadrado de Pearson $\left(X^{2}\right)$. Para explicar o efeito conjunto das variáveis preditoras sobre a variável dependente foi utilizada a Regressão de Logística Múltipla (RLM) com razão de chance ajustada (ORa).

Resultados: os participantes mais dispostos a receber uma vacina contra COVID-19 são os que se informaram sobre a mesma nas redes sociais (ORa = 4,56, IC 95\%: 1,77-11,72) e nos jornais e TV (ORa =2,74. IC95\%=1,07-7,04).

Conclusão: Ter informação sobre a vacina, seja por meio das redes sociais ou dos jornais e TV, aumentou a predisposição para tomá-la. Assim, se faz necessária a intensificação de informações efetivas sobre os benefícios das vacinas que serão aprovadas pela Agência Nacional de Vigilância Sanitária.

\section{Abstract}

Objective: To analyze COVID-19 vaccine acceptance among people diagnosed with flu-like illness.

Methods: This is a cross-sectional study with a random sample consisting of 454 participants. Data were collected from March to August 2020, in two stages: in the first, data available in the information systems were collected, using an instrument adapted from the investigation form of flu-like illness suspected by COVID-19, and in the second stage, primary data was collected from the participant through a telephone call. For the bivariate analysis, Pearson's chi-square test was performed $\left(X^{2}\right)$. To explain the joint effect of predictor variables on the dependent variable, Multiple Logistics Regression (MRL) with adjusted Odds Ratio (aOR) was used.

Results: Participants most willing to receive a COVID-19 vaccine are those who learned about it on social media (aOR $=4.56,95 \% \mathrm{Cl}: 1.77-11.72)$ and on newspapers and TV $(\mathrm{aOR}=2.74 .95 \% \mathrm{Cl}=1.07-7.04)$.

Conclusion: Having information about the vaccine, whether through social networks or newspapers and TV, increased the predisposition to take it. Thus, it is necessary to intensify effective information about the benefits of vaccines that will be approved by the Brazilian National Health Regulatory Agency (Agência Nacional de Vigilância Sanitária - ANVISA). 


\section{Resumen}

Objetivo: Analizar la aceptación de la vacuna contra la COVID-19 entre personas con diagnóstico de síndrome gripal.

Métodos: Estudio transversal con muestra aleatoria compuesta por 454 participantes. Los datos fueron recopilados en el período de marzo a agosto de 2020 , en dos etapas. En la primera, se recopilaron datos disponibles en los sistemas de información, utilizando instrumento adaptado de la ficha de investigación del síndrome gripal con sospecha de enfermedad por COVID-19; y en la segunda etapa, se realizó la recopilación del dato primario con el participante, por medio de llamada telefónica. En el análisis bivariado se realizó la prueba $\chi^{2}$ de Pearson. Para explicar el efecto conjunto de las variables predictoras sobre la variable dependiente, se utilizó la regresión logística múltiple (RLM) con razón de momios ajustada (ORa).

Resultados: Los participantes más dispuestos a recibir una vacuna contra la COVID-19 son los que se informaron sobre ella en las redes sociales (ORa = 4,56, IC 95 \%: 1,77-11,72) y en periódicos y por televisión (ORa =2,74. IC95 \%=1,07-7,04).

Conclusión: Tener información sobre la vacuna, ya sea por medio de las redes sociales o periódicos y televisión, aumentó la predisposición a recibirla. De esta forma, es necesario intensificar la información efectiva sobre los beneficios de las vacunas que serán aprobadas por la Agencia Nacional de Control Sanitario.

\section{Introdução}

A circulação do Novo Coronavírus (SARS-CoV-2) em âmbito mundial tem produzido uma crise sem precedentes, implicando em muitos enfrentamentos, em especial no setor saúde. COVID-19 refere-se à doença causada pelo SARS-CoV-2 que ocasiona diversas manifestaçóes clínicas, desde casos mais brandos a doenças graves envolvendo principalmente o sistema respiratório, com ocorrência de síndrome respiratória aguda grave, além de manifestaçôes multissistêmicas imunomediadas. ${ }^{(1,2)}$

Os primeiros casos da doença foram registrados na província Wuhan, na China, em dezembro de 2019 e a disseminação avançou nos três primeiros meses de 2020, de modo que até 26 de dezembro de 2020 já tinham sido registrados 80.293 .196 casos, em 220 países e 1.759 .457 mortes. ${ }^{(3)}$ A Organizaçáo Mundial da Saúde declarou a disseminaçáo da COVID-19 como pandemia em março de 2020, desde então, o seu combate tornou-se um desafio na comunidade científica em elucidar a progressão clínica da doença, terapias adequadas e o desenvolvimento de vacinas. ${ }^{(4)}$

No Brasil, o primeiro caso data de 26 de fevereiro de 2020 na cidade de Sáo Paulo, veiculado por um brasileiro vindo da Itália. A maior parte dos casos se concentra em Sáo Paulo, e, nos outros estados acredita-se que a contaminaçáo inicial também se apresentou por viajantes, sendo propagada em sua maior parte por transmissáo comunitária. Até 26 de dezembro de 2020, o país já contava com 7.448. 560 casos, 190.515 óbitos e 2,6\% de letalidade. No estado do Piauí, no mesmo período, havia o registro de 140.245 casos confirmados, distribuídos em 224 cidades, 2.802 óbitos e letalidade estimada em 2,2\%. Teresina na mesma data registrou 48.408 casos confirmados com 1.204 óbitos. ${ }^{(5,6)}$
Diante das incertezas que ainda cercam a COVID-19 e sem um tratamento eficaz que modifique o seu curso, a adoção de medidas de proteção é imprescindível no gerenciamento da pandemia e dentre estas, o desenvolvimento de vacinas é fundamental para o controle da infecção. A velocidade para o desenvolvimento de vacinas contra o SARSCoV-2 é inédita e uma variedade de plataformas tecnológicas têm sido testadas globalmente. ${ }^{(7)}$

De acordo com a OMS, até o dia 29 de dezembro de 2020, existiam 172 vacinas contra COVID-19 em desenvolvimento na fase pré-clínica e 60 vacinas candidatas em fase de pesquisa clínica. ${ }^{(8)} \mathrm{O}$ documento que operacionaliza o plano nacional brasileiro de vacinaçáo contra a COVID-19, considera como possíveis candidatas as 13 vacinas que se encontram em ensaios clínicos de fase III para avaliação de eficácia e segurança, e posterior imunização da populaçăo. ${ }^{(9)}$

A despeito do processo de vacinaçáo ser, em regra, revestido de segurança e eficácia, é crescente em escala mundial o fenômeno da hesitação vacinal, que se caracteriza pela recusa ou atraso em aceitar as vacinas. Existem alguns estudos ${ }^{(10-14)}$ sobre a aceitação da vacina contra a COVID-19, os quais apontam que países como China, Equador, Brasil, Malásia, Indonésia, Coréia do Sul, Sul da África, Dinamarca e Reino Unido, apresentam aceitaçáo variando entre 65 a 97\%. Por outro lado, a Rússia e França têm taxas de aceitação entre 55 a $62 \%$.

Discutir a aceitação das vacinas existentes é algo relevante, sobretudo no contexto da pandemia da COVID-19, considerando que esta pandemia foi acompanhada de uma infodemia, potencializada pelas redes sociais. O surgimento de rumores, informaçôes falsas e teorias de conspiraçáo têm causado desconfiança e insegurança sobre as vacinas. 
Assim, torna-se importante identificar a aceitação das pessoas em relação às vacinas, pois essas informaçóes são úteis para a definição de estratégias que melhorem a compreensão populacional e favoreça o controle da doença. ${ }^{(10)}$ Neste contexto, propôs-se desenvolver o primeiro estudo nacional que aborda a aceitação da vacina contra COVID-19, pois até o momento dados relacionados a esta questão foram publicados apenas em um estudo multinacional, que incluiu o Brasil. ${ }^{(13)}$ Dessa forma, objetiva-se com esse estudo, analisar a aceitabilidade da vacina contra COVID-19 entre o público com diagnóstico de síndrome gripal.

\section{Métodos}

Estudo transversal, realizado partir da base de dados de notificação de síndrome gripal suspeita de doença pelo Coronavírus. Desenvolvido no município de Teresina, cuja rede básica de saúde é composta por 91 Unidades Básicas de Saúde (UBS), das quais 73 na zona urbana e 18 situadas na zona rural, distribuídas em coordenadorias regionais de saúde (CRS): Norte, Sul, Leste e Sudeste. Adotou-se como critério de inclusão, as UBS que são referência para atendimento dos casos de síndrome gripal. Dentre as 91UBS, 20 atendiam a este critério, estando distribuídas da seguinte forma: 6 na CRS Sul; 5 CRS Norte; 6 na CRS Leste e 3 na CRS Sudeste.

A população fonte do estudo foi composta pelo universo dos casos com suspeita ou confirmaçáo de infecção pela COVID-19, atendidos e notificados nas UBS selecionadas $(n=20)$, no período de março de 2020, quando foi definida a transmissão comunitária do Coronavírus no Brasil, até agosto de 2020. Com relação ao tamanho da amostra, tomando-se por base prevalência presumida de $50 \%$, visto que não há dados na literatura a respeito, e este valor maximiza a amostra, ${ }^{(15)}$ erro tolerável de $5 \%$ e nível de confiança de $95 \%$, obteve-se o mínimo de 384 participantes. Aplicou-se uma taxa de $20 \%$ para recomposição, presumindo-se perdas durante a pesquisa. Considerando que sete foram excluídos pela falta de acesso aos seus telefones, a amostra final foi de 454 participantes.

Em um primeiro momento as UBS, foram relacionadas por área geográfica, conforme a localização do estabelecimento, enumeradas, e a partir dessa lista, foram organizadas nas quatro áreas das Coordenadorias Regionais de Saúde do município (Norte, Sul, Leste e Sudeste). Quanto à distribuição da amostra $(\mathrm{n}=454)$ nas UBS selecionadas, foi proporcional ao número de casos registrados nas mesmas. Após a distribuição proporcional da amostra, e de posse da listagem de pessoas atendidas nas UBS, procedeu-se ao sorteio dos participantes.

Ocorreu em duas etapas, na primeira, coletaram-se os dados disponíveis nos sistemas de informação, utilizando-se um instrumento adaptado da ficha de investigação de síndrome gripal suspeito de doença pelo Coronavírus - COVID-19, e na segunda etapa, procedeu-se a coleta do dado primário junto ao participante, por meio de ligação telefônica, com vista à complementação das informaçôes necessárias e a apresentação do Termo de Consentimento Livre e Esclarecido (TCLE).

As variáveis do estudo são: Idade, escolaridade, renda, sexo, raça, profissão, comorbidades, teste para COVID, interesse em tomar a vacina contra COVID-19, motivo do não interesse, e fonte de informação sobre a vacina. $\mathrm{O}$ interesse em ser vacinado contra a COVID-19 (variável desfecho) foi medido com a pergunta: "Quando uma vacina contra COVID-19 estiver disponível, você aceita ser vacinado? As opçóes de resposta eram "sim", "não". Os participantes que responderam "não" foram solicitados a fornecer um motivo. Também se perguntou qual a fonte que usavam para se informar sobre a vacina.

Os dados foram analisados com a utilização do aplicativo Statistical Package for the Social Science (SPSS), versão 22.0. Para a análise univariada foi usada a distribuição absoluta e percentual. $\mathrm{Na}$ análise bivariada foi utilizado o teste qui-quadrado de Pearson $\left(\mathrm{X}^{2}\right)$, para associar as variáveis quantitativas explicativas com as variáveis respostas do estudo: Interesse em se vacinar, motivo do não interesse na vacina e fonte de informação sobre a vacina. Para explicar o efeito conjunto das variáveis preditoras sobre a variável dependente foi utilizado a Regressão de Logística Múltipla (RLM) com razão de chance ajustada (ORa). O critério para inclusão de variáveis no modelo logístico foi a associação ao nível de $20 \%$ $(\mathrm{p}<0,200)$ na análise bivariada. ${ }^{(16-18)} \mathrm{O}$ critério de significância ou permanência das variáveis no modelo, por sua vez, foi a associação em nível de $5 \%(\mathrm{p}<0,05)$. 
Tabela 1. Análise Bivariada do interesse na vacina contra COVID-19 segundo as variáveis sociodemográficas e resultado de teste para COVID-19

\begin{tabular}{|c|c|c|c|c|}
\hline \multirow{2}{*}{ Variáveis } & & \multicolumn{2}{|c|}{ Interesse na vacina } & \multirow[b]{2}{*}{$p$-value ${ }^{\star}$} \\
\hline & & $\begin{array}{l}\text { Não } \\
\mathrm{n}(\%)\end{array}$ & $\begin{array}{l}\text { Sim } \\
\mathrm{n}(\%)\end{array}$ & \\
\hline \multirow[t]{2}{*}{ Sexo } & Masculino & $58(21,9)$ & $207(78,1)$ & 0,381 \\
\hline & Feminino & $35(18,5)$ & $154(81,5)$ & \\
\hline \multirow[t]{4}{*}{ Raça/Cor } & Branca & $29(27,9)$ & $75(72,1)$ & 0,189 \\
\hline & Parda & $49(18,8)$ & $211(81,2)$ & \\
\hline & Amarela & $3(15,0)$ & $17(85,0)$ & \\
\hline & Preta & $12(17,1)$ & $58(82,9)$ & \\
\hline \multirow[t]{2}{*}{ Idade } & Menos de 60 anos & $80(20,2)$ & $316(79,8)$ & 0,697 \\
\hline & Maior ou igual a 60 anos & $13(22,4)$ & $45(77,6)$ & \\
\hline \multirow[t]{4}{*}{ Escolaridade } & Não alfabetizado & $5(27,8)$ & $13(72,2)$ & 0,437 \\
\hline & Fundamental & $11(15,5)$ & $60(84,5)$ & \\
\hline & Médio & $44(19,5)$ & $182(80,5)$ & \\
\hline & Superior & $33(23,7)$ & $106(76,3)$ & \\
\hline \multirow{4}{*}{$\begin{array}{l}\text { Renda (em salário- } \\
\text { mínimo) }\end{array}$} & Menos de 1 & $14(19,2)$ & $59(80,8)$ & 0,339 \\
\hline & 1 a menos de 2 & $29(16,7)$ & $145(83,3)$ & \\
\hline & 2 a 4 & $24(23,8)$ & $77(76,2)$ & \\
\hline & Mais de 4 & $26(24,5)$ & $80(75,5)$ & \\
\hline \multirow{2}{*}{$\begin{array}{l}\text { Profissional de } \\
\text { saúde }\end{array}$} & Não & $157(38,1)$ & $255(61,9)$ & 0,003 \\
\hline & Sim & $8(18,7)$ & $34(81,3)$ & \\
\hline \multirow{2}{*}{$\begin{array}{l}\text { Teste para } \\
\text { COVID-19 }\end{array}$} & Positivo & $67(24,0)$ & $212(76,0)$ & 0,019 \\
\hline & Negativo & $26(14,9)$ & $149(85,1)$ & \\
\hline \multirow{4}{*}{$\begin{array}{l}\text { Fonte de informação } \\
\text { sobre a vacina }\end{array}$} & Redes sociais & $22(12,4)$ & $156(87,6)$ & $<0,001$ \\
\hline & Profissional de saúde & $40(32,3)$ & $84(67,7)$ & \\
\hline & Jornal/TV & $22(17,3)$ & $105(82,7)$ & \\
\hline & Não buscou informação & $9(36,0)$ & $16(64,0)$ & \\
\hline
\end{tabular}

${ }^{*} p$-value - significância estatística foi fixada em $p \leq 0,05$
A pesquisa foi executada após aprovação do Comitê de Ética e Pesquisa (CEP), da Universidade Federal do Piauí. Respeitaram-se os preceitos éticos contidos na Resolução 466/2012, do Conselho Nacional de Saúde (CAAE: 33801920.4.0000.5214).

\section{Resultados}

O estudo foi conduzido com 454 participantes, com idade de 18 a 88 anos de idade, e predominância do sexo masculino $(60,5 \%)$, raça/cor parda $(57,3 \%)$ e escolaridade superior (49,8\%). Em relação a inserção no mercado de trabalho, 65,7\% possuíam emprego. A renda declarada variou de menos de um salário-mínimo (38,3\%) até mais de quatro (23,3\%). Quanto à categoria profissional, 9,3\% eram trabalhadores da saúde e $90,7 \%$ eram de outras profissóes (Tabela 1). Verificou-se que 79,5\% (361) dos participantes manifestaram-se favoráveis à vacina contra COVID-19. O interesse pela vacina foi associado a ser profissional de saúde $(\mathrm{p}=0,003)$, ser testado para a COVID-19 $(0,019)$ e buscar fontes de informação sobre a vacina $(\mathrm{p}<0,001)$ (Tabela 1$)$.

Na tabela 2, observam-se os fatores envolvidos na decisão de não tomar a vacina contra COVID-19:

Tabela 2. Análise Bivariada dos motivos do não interesse em se vacinar contra a COVID-19 segundo as das variáveis sociodemográficas e informações sobre a vacina

\begin{tabular}{|c|c|c|c|c|c|c|c|}
\hline \multirow{3}{*}{ Variáveis } & & \multicolumn{6}{|c|}{ Motivo do não interesse em se vacinar } \\
\hline & & \multicolumn{3}{|c|}{ Medo de EAPV* } & \multicolumn{3}{|c|}{ Desconfiança } \\
\hline & & $\begin{array}{l}\text { Não } \\
\mathrm{n}(\%)\end{array}$ & $\begin{array}{c}\text { Sim } \\
\mathrm{n}(\%)\end{array}$ & $\mathrm{p}$-value ${ }^{\dagger}$ & $\begin{array}{l}\text { Não } \\
\mathrm{n}(\%)\end{array}$ & $\begin{array}{l}\text { Sim } \\
\mathrm{n}(\%)\end{array}$ & p-value ${ }^{\dagger}$ \\
\hline \multirow{2}{*}{ Sexo } & Masculino & $29(50,0)$ & $29(50,0)$ & 0,689 & $28(48,3)$ & $30(51,7)$ & 0,437 \\
\hline & Feminino & $16(45,7)$ & $19(54,3)$ & & $14(40,0)$ & $21(60,0)$ & \\
\hline \multirow{4}{*}{ Raça } & Branca & $14(48,3)$ & $15(51,7)$ & 0,231 & $13(44,8)$ & $16(55,2)$ & 0,229 \\
\hline & Parda & $24(49,0)$ & $25(51,0)$ & & $22(44,9)$ & $27(55,1)$ & \\
\hline & Amarela & $3(100,0)$ & - & & $3(100,0)$ & - & \\
\hline & Preta & $4(33,3)$ & $8(66,7)$ & & $4(33,3)$ & $8(66,7)$ & \\
\hline \multirow{2}{*}{ Idade } & Menos de 60 anos & $40(50,0)$ & $40(50,0)$ & 0,441 & $37(46,3)$ & $43(53,8)$ & 0,601 \\
\hline & Maior ou igual a 60 anos & $5(38,5)$ & $8(61,5)$ & & $5(38,5)$ & $8(61,5)$ & \\
\hline \multirow{4}{*}{ Escolaridade } & Não alfabetizado & $15(45,5)$ & $18(54,5)$ & 0,059 & $4(80,0)$ & $1(20,0)$ & 0,252 \\
\hline & Fundamental & $5(100,0)$ & - & & $3(27,3)$ & $8(72,7)$ & \\
\hline & Médio & $3(27,3)$ & $8(72,7)$ & & $21(47,7)$ & $23(52,3)$ & \\
\hline & Superior & $22(50,0)$ & $22(50,0)$ & & $14(42,4)$ & $19(57,6)$ & \\
\hline \multirow{4}{*}{$\begin{array}{l}\text { Renda (em salário- } \\
\text { mínimo) }\end{array}$} & Menos de 1 & $6(42,9)$ & $8(57,1)$ & 0,242 & $6(42,9)$ & $8(57,1)$ & 0,492 \\
\hline & 1 a menos de 2 & $12(41,4)$ & $17(58,6)$ & & $11(37,9)$ & $18(62,1)$ & \\
\hline & 2 a 4 & $10(41,7)$ & $14(58,3)$ & & $10(41,7)$ & $14(58,3)$ & \\
\hline & Mais de 4 & $17(65,4)$ & $9(34,6)$ & & $15(57,7)$ & $11(42,3)$ & \\
\hline \multirow{2}{*}{ Profissional de saúde } & Sim & $9(56,3)$ & $7(43,8)$ & 0,489 & $9(56,3)$ & $7(43,8)$ & 0,327 \\
\hline & Não & $36(46,8)$ & $41(53,2)$ & & $33(42,9)$ & $44(57,1)$ & \\
\hline \multirow{3}{*}{$\begin{array}{l}\text { Fonte de informação } \\
\text { sobre a vacina }\end{array}$} & Redes sociais & $12(54,5)$ & $10(45,5)$ & 0,834 & $11(50,0)$ & $11(50,0)$ & 0,764 \\
\hline & Profissional de saúde & $20(50,0)$ & $20(50,0)$ & & $20(50,0)$ & $20(50,0)$ & \\
\hline & Jornal/TV & $10(45,5)$ & $12(54,5)$ & & $9(40,9)$ & $13(59,1)$ & \\
\hline
\end{tabular}

*EAPV - evento adverso pós vacinação; ${ }^{\dagger} p$-value - significância estatística foi fixada em $p \leq 0,05$ 
evento adverso pós vacinação e desconfiança quanto a seriedade no processo de fabricação da vacina. Nenhum dos dois motivos foi estatisticamente associado às características sociodemográficas e nem a fonte de informação dos participantes.

Dentre as três variáveis que atenderam aos requisitos do modelo multivariado, como preditora do interesse em tomar a vacina contra a COVID-19, a fonte de informação, mediante o acesso pelas redes sociais, aumentou em 4,56 vezes as chances de tomar a vacina, bem como informação por meio de jornal e TV, que aumentou em 2,74. Ser profissional de saúde mostrou-se associado ao interesse em receber a vacina, porém, não houve significância estatística (Tabela 3).

Tabela 3. Regressão Logística do interesse em receber a vacina contra a COVID-19 segundo a categoria profissional, testes para COVID-19 e fonte de informação sobre a vacina

\begin{tabular}{|c|c|c|c|c|c|}
\hline \multirow{2}{*}{ Variáveis } & & \multirow{2}{*}{ ORa* } & \multicolumn{2}{|c|}{ IC $95 \%^{\dagger}$} & \multirow{2}{*}{$p$-value } \\
\hline & & & $\mathrm{Ll}^{\ddagger}$ & $\mathrm{LS}^{\S}$ & \\
\hline \multirow{2}{*}{$\begin{array}{l}\text { Profissional de } \\
\text { saúde }\end{array}$} & Não & 1 & & & 0,086 \\
\hline & Sim & 1,90 & 0,91 & 3,93 & \\
\hline \multirow{3}{*}{$\begin{array}{l}\text { Teste para } \\
\text { COVID-19 }\end{array}$} & Positivo & 0,67 & 0,38 & 1,16 & 0,153 \\
\hline & Negativo & 1 & & & \\
\hline & Redes sociais & 4,56 & 1,77 & 11,72 & 0,002 \\
\hline \multirow{3}{*}{$\begin{array}{l}\text { Fonte de } \\
\text { informação sobre } \\
\text { a vacina }\end{array}$} & $\begin{array}{l}\text { Profissional de } \\
\text { saúde }\end{array}$ & 1,62 & 0,63 & 4,17 & 0,314 \\
\hline & Jornal/TV & 2,74 & 1,07 & 7,04 & 0,036 \\
\hline & $\begin{array}{l}\text { Não buscou } \\
\text { informação }\end{array}$ & 1 & & & \\
\hline
\end{tabular}

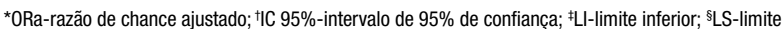
superior; ' $p$-value - significância estatística foi fixada em $p \leq 0,05$

\section{Discussão}

O estudo revelou que a expressiva maioria dos adultos estaria disposta em tomar a vacina contra COVID-19, resultado que coaduna com os estudos publicados sobre a aceitabilidade da vacina. ${ }^{(19-23)}$ Cabe destacar, que este achado representa uma estimativa de aceitabilidade da vacina contra COVID-19 e pode ser usado para orientar o planejamento e as estratégias de comunicação para aumentar a adesão à campanha da vacinação, quando a vacina estiver disponível. A aceitabilidade da vacina foi mais prevalente entre os profissionais de saúde, entre os que apresentaram teste para COVID-19 negativo e entre aqueles que buscaram informaçóes sobre a vacina nas redes sociais e jor-
nal/TV, sendo estatisticamente associada à busca de informação.

Nos Estados Unidos, estudo desenvolvido para compreender as atitudes e obstáculos frente à vacinação contra COVID-19, evidenciou que aproximadamente $68 \%$ de todos os participantes apoiaram a vacinação, mas os efeitos colaterais, eficácia e a duração dos testes continuou sendo uma preocupação. Testes mais longos, maior eficácia foram significativamente associados ao aumento da aceitação da vacina. Promoção de campanhas informativas sobre a vacinação contra COVID-19 deve esclarecer as preocupações daqueles que já estão hesitantes com vacina, e se direcionar aos benefícios da vacinação para o país. Deve-se dedicar tempo suficiente para dissipar as preocupaçóes sobre os efeitos colaterais antes do lançamento da vacina. ${ }^{(24)}$

Outro estudo realizado nos Estados Unidos, que investigou a probabilidade do participante de selecionar e receber uma vacina hipotética, verificou que $79 \%$ dos participantes selecionaram uma das vacinas hipotéticas e $21 \%$ não selecionaram nenhuma das vacinas. Os principais atributos da vacina para a seleção e disposição em recebê-la foi o aumento da eficácia, seguido por uma maior duração de proteção e menor incidência de eventos adversos. Os participantes eram menos propensos a selecionar uma vacina desenvolvida fora dos Estados Unidos, particularmente da China, ou uma vacina aprovada via autorização de uso de emergência do Food and Drug Administration (FDA). O endosso do Centro de Controle e Prevenção de Doenças (CDC) e da Organização Mundial de Saúde (OMS) foram associados a maiores probabilidades de seleção de vacina. ${ }^{(21)}$ Estes resultados se assemelham aos deste estudo, cuja prevalência de aceitação da vacina também chegou a aproximadamente $80 \%$ e os possíveis eventos adversos pós-vacinação, se constituem em um dos motivos da não aceitação.

Levantamento conduzido na Arábia Saudita que investigou a predisponibilidade de aceitar a futura vacina contra COVID-19, observou que 64,7\% disseram sim para tomar a vacina COVID-19, 7,0\% disseram não, e 28,2\% disseram não tenho certeza. Além disso, os idosos e casados são mais propensos a aceitar a vacina contra COVID-19. A confiança dos 
participantes do estudo no sistema de saúde e o risco percebido de adquirir infecção foram considerados preditores significativos para explicar a aceitaçáo da vacina contra COVID-19. ${ }^{(25)}$ Contrariamente, neste estudo não foi observada nenhuma associação entre o interesse de receber a vacina e os dados sociodemográficos dos participantes.

No Reino Unido, pesquisa conduzida para investigar os fatores associados à intenção de ser vacinado contra COVID-19, apesar da incerteza em torno dos detalhes de uma vacinaçáo, a maioria dos participantes relatou a intenção de ser vacinada contra COVID-19, 64\% apresentaram grande probabilidade de serem vacinados, $27 \%$ náo tinham certeza e $9 \%$ relataram ser muito improvável concordarem em ser vacinado. A intenção de ser vacinado foi associada a crenças e atitudes gerais sobre a vacinação contra COVID-19, crenças mais fracas de que a vacinação causaria efeitos adversos ou seria insegura, maior percepção de suficiência de informações para tomar uma decisão informada sobre vacinação, maior percepção de risco de COVID-19, idade avançada, e ter sido vacinado contra a gripe no passado. A população precisa ser informada sobre a importância de vacinar todas as pessoas que compóem os grupos prioritários e sobre o potencial risco da COVID-19 continuar com alta circulação, se um significativo contingente populacional não se vacinar. ${ }^{(26)}$

Nesta perspectiva, o envolvimento dos profissionais de saúde, especialmente daqueles inseridos na atenção primária à saúde, em ações estratégicas que levem informaçóes seguras, assertivas e objetivas à população, são de fundamental importância. Destaca-se que é necessário os profissionais de saúde, estarem suficientemente capacitados, porque as seus conhecimentos e atitudes frente às vacinas são um determinante importante de sua própria adesão à vacina e da probabilidade de recomendar a vacina aos seus pacientes. ${ }^{(27)}$

Observou-se que um grande percentual de profissionais de saúde $(81,3 \%)$, se mostrou favorável à vacinação, contrariando o estudo realizado no Congo, em que apenas $27,7 \%$ dos profissionais de saúde, disseram que aceitariam uma vacina contra COVID-19 se estivesse disponível. ${ }^{(21)}$ Muitos fatores podem estar associados à predisposiçáo para aceitar a vacina. A alta taxa de infecção pela COVID-19, independente da taxa de letalidade estar alta ou não, a falta de tratamento com base em fortes evidências científicas, o risco individual percebido, a estrutura insuficiente dos serviços para atendimento aos casos mais graves e a alta eficácia das vacinas que estão sendo produzidas ilustram as condições circunstanciais no contexto da pandemia, que favorecem as atitudes favoráveis à sua aceitação. ${ }^{(20)}$

Em cada local em que as pesquisas foram desenvolvidas, os fatores podem diferir dependendo do contexto local e como a vacina e as demais medidas de prevenção estão sendo conduzidas. Destaca-se, portanto, a importância de utilizar-se dos meios de comunicação para intensificar as informaçóes sobre a vacina contra COVID-19, adequando-as, com foco nas questóes como eficácia da vacina, duração da proteção, esquema, vacinação e recomendação dos serviços de saúde, além de esclarecimentos sobre os eventos adversos pós vacinação e sobre o processo de fabricação da vacina.

Observou-se nesta pesquisa, que entre os participantes do estudo que ainda se mantêm hesitantes em aceitar a vacina, os fatores relacionados aos eventos adversos pós vacinação e a desconfiança quanto a seriedade no processo de fabricação da vacina foram predominantes, corroborando outros estudos sobre a aceitabilidade da vacina, publicados em diferentes países. Ressalta-se que os motivos de hesitação vacinal identificados, pode dever-se ao momento em que se realizou a pesquisa.

Estudo conduzido nos Estados Unidos identificou que apenas cerca de seis em cada dez entrevistados disseram sim, quando questionados se eles serão vacinados quando uma vacina contra COVID-19 estiver disponível. Contudo, $10 \%$ relataram que não receberiam a vacina, por não acreditar em vacinas, não querer a vacina, não se sentirem confortáveis com ela, além da preocupação com segurança, efeitos colaterais e desconfiança geral. Esse grupo pode ser muito difícil de ser persuadido a vacinar-se e informaçôes consistentes devem ser conduzidas com atenção, devido ao fortalecimento do movimento antivacina e contínua politização da vacina. Acrescenta-se ainda o grupo que compóe o movimento de hesitação em torno da vacina contra COVID-19, cujo percentual é em torno de $30 \%$. As suas razóes giram em torno 
da segurança, eficácia eforte necessidade de mais informaçóes sobre a vacina. ${ }^{(28)}$

As atitudes negativas em relação à vacinação geral, em função dos eventos adversos pós-vacinação e dos possíveis danos a longo prazo, contribuíram de forma independente para a hesitação em aceitar a vacina contra COVID-19. Os relatos de eventos adversos de alguns à vacina testada pode abalar ainda mais a confiança das pessoas na vacina. ${ }^{(29)}$

Diante da hesitação de alguns em ser vacinado, é necessário estabelecer uma comunicação efetiva com a população, ressaltando alguns aspectos relacionados à importância da vacinação, a segurança e eficácia das vacinas e sobre a necessidade de manter as medidas não farmacológicas de prevenção, mesmo após a vacinação, além de buscar combater as fake news. ${ }^{(25)}$

Uma limitação do estudo é o corte transversal, que não possibilita o acompanhamento da decisão de aceitar ou não a vacina, entre os participantes. Acrescenta-se o fato de que a coleta de dados ocorreu durante as fases iniciais de desenvolvimento de uma vacina COVID-19, não sendo possível fornecer aos participantes informaçóes sobre a mesma, que podem afetar a aceitabilidade.

\section{Conclusão}

O estudo fornece uma visão inicial sobre a aceitabilidade de uma vacina COVID-19, com resultados indicando que um alto percentual $(79,5 \%)$ de adultos estaria disposto a ser vacinado. Os participantes mais predispostos a receber uma vacina contra COVID-19 são os que se informaram sobre a vacina nas redes sociais ou jornal/TV. Assim, recomenda-se que campanhas informativas sobre os benefícios das vacinas que serão aprovadas pela Agência Nacional de Vigilância Sanitária sejam realizadas.

\section{Agradecimentos}

Aos enfermeiros: Francisca Gomes da Cruz, Antonio Mariano da Costa Neto e Soraia Martins da Fonseca, que contribuíram com as ligaçóes por telefone para os participantes do estudo.

\section{Colaborações}

Araújo TME, Carvalho AMC, Fronteira I, Silva AAS, Rodrigues KA, Queiroz GS e Carcará LRA contribuíram com a concepção do estudo, análise e interpretação dos dados, redação do artigo, revisão crítica relevante do conteúdo intelectual e aprovação da versão final a ser publicada.

\section{Referências}

1. Adhikari SP, Meng S, Wu YJ, Mao YP, Ye RX, Wang QZ, et al. Epidemiology, causes, clinical manifestation and diagnosis, prevention and control of coronavirus disease (COVID-19) during the early outbreak period: a scoping review. Infect Dis Poverty. 2020;9(1):29. Review.

2. Hanff TC, Harhay MO, Brown TS, Cohen JB, Mohareb AM. Is there an association between COVID-19 mortality and the renin-angiotensin system? a call for epidemiologic investigations. Clin Infect Dis. 2020;71(15):870-4.

3. Worldometer. Coronavirus. COVID-19 coronavirus pandemic United States: Worldometer; 2020 [cited 2020 Dec 26]. Available from: https://www.worldometers.info/coronavirus

4. Schaefer GO, Tam CC, Savulescu J, Voo TC. COVID-19 vaccine development: time to consider SARS-CoV-2 challenge studies? Vaccine. 2020;38(33):5085-8.

5. Brasil. Ministério da Saúde. Painel Coronavírus, 2020. Brasília (DF): Ministério da Saúde; 2021 [citado 2020 Dez 26]. Disponível em: https://covid.saude.gov.br/

6. Piauí. Governo do Estado do Piauí. Painel Epidemiológico Covid-19. Piauí: Governo do Estado do Piauí [citado 2020 Dez 26]. Disponível em: https://www.pi.gov.br/coronavirus/

7. Arora NK, Das MK. COVID-19 vaccine development and the way forward. Indian J Public Health. 2020;64(2):S108-11.

8. World Health Organization (WHO). Draft landscape of COVID-19 candidate vaccines. Genève: WHO; 2020 [cited 2021 Jan 5]. Available from: www.who.int/publications/m/item/draft-landscape-of-covid-19candidate-vaccines

9. Brasil. Ministério da Saúde. Secretaria de Vigilância em Saúde. Departamento de Imunização e Doenças Transmissíveis. Coordenação-Geral do Programa Nacional de Imunizações. Plano nacional de operacionalização da vacinação contra a COVID-19. Brasília (DF): Ministério da Saúde; 2020 [citado 2021 Jan 4]. Disponível em: https://www.gov.br/saude/pt-br/media/pdf/2020/ dezembro/17/17-12-2020_plano_vacinacao_covid19_versao_ eletronica.pdf

10. Reiter PL, Pennell ML, Katz ML. Acceptabilityof a COVID-19 vaccineamongadults in the United States: How many people would get vaccinated? Vaccine. 2020;38(42):6500-7.

11. Wang J, Jing R, Lai $X$, Zhang $H$, Lyu $Y$, Knoll MD, et al. Acceptance of COVID-19 Vaccination during the COVID-19 Pandemic in China. Vaccines. 2020;8(3):482.

12. Sonawane K, Troisi CL, Deshmukh AA. COVID-19 vaccination in the UK: addressing vaccine hesitancy. Lancet Reg Health Eur. $2021 ; 1: 100016$ 
13. Lazarus JV, Ratzan SC, Palayew A, Gostin LO, Larson HJ, Rabin K, et al. A global survey of potential acceptance of a COVID-19 vaccine. Nat Med. 2021;27(2):225-8. Erratum in: Nat Med. 2021 Jan 11.

14. Schwarzinger M, Watson V, Arwidson P, Alla F, Luchini S. COVID-19 vaccine hesitancy in a representative working-age population in France: a survey experiment based on vaccine characteristics. Lancet Public Health. 2021;6(4):e210-21.

15. Hulley SB, Cummigs SR, Browner WS, Grady DG, Newman TB. Delineando a pesquisa clínica. 4 ed. Porto Alegre: Artmed; 2015.

16. Armitage P, Berry G, Matthews JN. Statistical methods in medical research. 4th ed. Nova Jersey: Wiley; 2002.

17. Pestana H, Gageiro J. Data analysis for social sciences: the complementarity of SPSS. 6 ed. Lisboa: Edicoes Silabo; 2014.

18. Hosmer DW, Lemeshow S. Applied Logistic Regression. 2nd ed. Nova Jersey: Wiley; 2000.

19. Detoc M, Bruel S, Frappe P, Tardy B, Botelho-Nevers E, Gagneux-Brunon A. Intention to participate in a COVID-19 vaccine clinical trial and to get vaccinated against COVID-19 in France during the pandemic. Vaccine. 2020;38(45):7002-6.

20. García LY, Cerda AA. Acceptance of a COVID-19 vaccine: a multifactorial consideration. Vaccine. 2020;38(48):7587.

21. Kreps S, Prasad S, Brownstein JS, Hswen Y, Garibaldi BT, Zhang B, et al. Factors Associated With US Adults' LikelihoodofAccepting COVID-19 Vaccination. JAMA Netw Open. 2020;3(10):e2025672.

22. Sarasty 0 , Carpio CE, Hudson D, Guerrero-Ochoa PA, Borja I. The demand for a COVID-19 vaccine in Ecuador. Vaccine. 2020;38(51):8090-8.
23. Herrera-Añazco $P$, Uyen-Cateriano A, Urrunaga-Pastor D, BendezuQuispe G, Toro-Huamanchumo CJ, Rodriguez-Morales AJ, et al. Prevalencia y factores asociados a la intención de vacunación contra la COVID-19 enel Perú. SciELO Preprints. 2021 Feb 23. D0I: 10.1590/ SciELOPreprints. 1879

24. Pogue K, Jensen JL, Stancil CK, Ferguson DG, Hughes SJ, Mello EJ, et al. Influences on Attitudes Regarding Potential COVID-19 Vaccination in the United States. Vaccines (Basel). 2020;8(4):582.

25. Billon-Denis E, Tournier JN. COVID-19 et vaccination: une dérégulation globale. Med Sci. 2020;36(11):1034-7.

26. Sherman SM, Smith LE, Sim J, Amlôt R, Cutts M, Dasch H, et al. COVID-19 vaccination intention in the UK: results from the COVID-19 vaccination acceptability study (CoVAccS), a nationally representative cross-sectional survey. Hum Vaccin Immunother. 2021;17(6):1612-21.

27. Kabamba Nzaji M, Kabamba Ngombe L, Ngoie Mwamba G, Banza Ndala DB, Mbidi Miema J, Luhata Lungoyo C, et al. Acceptability of vaccination against COVID-19 Among Healthcare Workers in the Democratic Republic of the Congo. Pragmat Obs Res. 2020;11:1039.

28. Fisher KA, Bloomstone SJ, Walder J, Crawford S, Fouayzi H, Mazor KM. Attitudes toward a potential sars-cov-2 vaccine: a survey of U.S. Adults. Ann Intern Med. 2020;173(12):964-73.

29. Qiao S, Tam CC, LiX. Risk exposures, risk perceptions, negative attitudes toward general vaccination, and COVID-19 vaccine acceptance among college students in South Carolina. MedRxiv [Preprint]. 2020 Nov 30:2020.11.26.20239483. Update in: Am J Health Promot. 2021 Jun 24. 